\title{
Variations
}

Variations

Revue internationale de théorie critique

$20 \mid 2017$

Expériences oppositionnelles

\section{Le corporatisme à la française}

Un anti-mouvement social ou une institution inoxydable?

\section{Bruno Lefebvre}

\section{(2) OpenEdition}

Journals

Édition électronique

URL : http://journals.openedition.org/variations/803

DOI : 10.4000 /variations.803

ISSN : 1968-3960

Éditeur

Les amis de Variations

Référence électronique

Bruno Lefebvre, "Le corporatisme à la française », Variations [En ligne], 20 | 2017, mis en ligne le 25 avril 2017, consulté le 25 février 2021. URL : http://journals.openedition.org/variations/803 ; DOI :

https://doi.org/10.4000/variations.803

Ce document a été généré automatiquement le 25 février 2021.

Les ami•e•s de Variations 


\title{
Le corporatisme à la française
}

\author{
Un anti-mouvement social ou une institution inoxydable?
}

\author{
Bruno Lefebvre
}

1 Au début du XXe siècle, avec les diffusions technologiques et l'invention du tourisme balnéaire et Alpin pour l'aristocratie et la bourgeoisie, le monde politique français s'interroge sur une réforme des structures dans l'organisation économique et sociale, le XIX ${ }^{e}$ siècle chaotique ayant laissé des mauvais souvenirs avec ses guerres, révoltes, grèves et changements de régimes incessants. En 1911, F. Millerand (1859/1943), Ministre des Finances et la Guerre, demande au sociologue E. Durkheim ${ }^{1}$ d'imaginer de $^{2}$ nouvelles institutions. Durkheim est obsédé à l'époque par la Morale, l'ordre, une solidarité qui respecte l'individualisme et les deux hommes mettent au point le projet d'un Parlement professionnel qui serait l'égal d'une chambre des députés et qui fonctionnerait en parallèle pour gérer l'économie et la production nationale. Le sociologue plaide pour un rétablissement des corporations style "Ancien Régime ", chaque activité professionnelle élisant ses représentants et disposant du monopole légal de ses pratiques et activités, légiférant de manière interne, organisant les enseignements, grades, rémunérations de manière autonome. Ainsi, le sentiment de Fraternité, de solidarité, de la défense des intérêts communs serait-il généré pour le bien de tous.

2 Après la guerre «scientifique » de 1914/18, ces idées de corporatisme s'emparent des mouvements sociaux autour des CGT, CGT-U, CFTC qui se structurent en «branches professionnelles ", c'est le moment où l'on pense des maisons du peuple et que l'on aspire aux loisirs. Les syndicats interviendraient dans l'évaluation des compétences des salariés, leurs formations et promotions. Du côté de la droite, Millerand a promu le modèle, en opposition avec les socialistes des années 1920. Les mouvements populistes français, comme en Hongrie, Pologne, Espagne, Portugal, Italie, Belgique et Roumanie à la même époque, mais chacun avec leurs variantes nationales, infiltrent cette organisation ou ce modèle d'organisation à peine institué. L'émiettement des forces professionnelles qui pourrait en résulter est contrecarré par notre célèbre sociologue national, Gustave Le Bon ${ }^{2}$ (hélas guère enseigné dans les universités françaises) qui tient salon à Paris, entretient des correspondances avec Benito Mussolini, et a comme 
élève Joseph Goebbels : les foules, impressionnables, peuvent s'unir, faire fusion, pour dépasser les clivages professionnels. Les nouvelles technologies comme la radio ou le cinéma sont pensées comme des outils d'unification.

L'extrême droite représentée par Charles Maurras ${ }^{3}$ (1868/1952), théoricien, pense la mise en place des corporations professionnelles réunies en faisceaux, terme qui est aussi à la racine du mot "fasciste». Après discussions avec mes vieux collègues syndicalistes en Europe, on se rend compte que les pays ayant connu un régime fasciste, France, Italie, Espagne, Portugal, ont des CGT respectivement identiques du point de vue organisationnel, en "branche ", à la différence des pays germaniques, anglo-saxons ou scandinaves.

Des syndicats professionnels et corporatistes, qui existent encore aujourd'hui, sont mis en place pendant les années 1930 avec caisses d'assurance, de retraite, camps de vacances. J'ai eu l'occasion, lorsque je travaillai sur «les routiers", de rencontrer, en 1987 (il est décédé peu après), un monsieur jovial qui a fondé l'un de ces syndicats et qui m'a fait l'honneur de me recevoir une semaine dans le bureau national où j'ai pu lire tous les journaux, photocopier et discuter. Je l'en remercie grandement. L'homme, admirateur de Mussolini, est blessé par une grenade lors de la guerre d'Ethiopie. Rentré en France, il crée un syndicat, un journal, une petite administration et devient journaliste. Pendant le régime de Philippe Pétain, il travaille au Ministère de l'information. Il se cache entre 1945 et 1947 pour ne pas être enquêté par la justice et les comités de libération, puis, profitant de ses relations, il crée les « $24 \mathrm{~h}$ du Man » où se côtoient de manière discrète, comme j'ai pu l'observer, étant introduit de manière « ethnographique », personnel politique, financier, vedettes du show-business, pilotes, industriels de l'automobile, équipes salariées des entreprises du secteur.

Pendant le régime de Vichy sont mises en place nombre d'institutions et juridictions encore actuelles et fonctionnelles aujourd'hui. Sur le modèle corporatiste, on crée les Ordres, des médecins, notaires, avocats, architectes, journalistes (le projet est malin : l'ordre des journalistes peut attaquer en justice quiconque délivre des informations sans avoir prêté serment à l'ordre, tout comme l'ordre des médecins peut vous attaquer en justice pour « exercice illégal de la médecine »). Nous devons également à ce modèle la FNSEA, syndicat issu de la corporation agricole qui s'occupe des assurances sociales et des retraites, de la transmission des sols, de la répartition des subventions européennes aux agriculteurs et qui nomme les Ministres de l'Agriculture depuis trois quarts de siècle.

6 Les fonctionnaires de ce gouvernement de Vichy, en tant que serviteurs de l'Etat, se reconvertissent évidement dans les institutions de la IV ${ }^{e}$ puis $V^{e}$ République. Du point de vue de ces serviteurs de l'Etat, le fait de passer d'un régime à un autre, n'est pas une contradiction, tout comme changer d'étiquette politique, et la population française l'accepte largement ${ }^{4}$; l'exemple le plus connu est celui de François Mitterrand: haut fonctionnaire dans les années 1940, Ministre de l'Intérieur pendant la guerre d'Algérie ayant condamné à la guillotine 50 personnes environ, puis promoteur de l'abolition de la peine de mort en tant que Président de la République. Les fonctionnaires des années 1940 investissent donc les syndicats, les nouvelles administrations, les comités d'entreprise et mettent en place avec les communistes, la CGT et le Conseil National de la Résistance, les régimes généraux de la Sécurité Sociale, les couvertures santé et le régime général des retraites. Comme nous le constatons, les nouvelles générations de " décideurs ", nées à partir des années 1940, élus de la République ou dans les Conseils 
d'Administrations des firmes transnationales, sont peu sympathisants du travail de leurs aînés et les régimes sociaux sont détricotés.

7 Les mouvements sociaux ne vont donc pas être issus par la suite de cette génération. La guerre «froide » USA/URSS va structurer pendant cette période les opinions et le syndicalisme se divise avec la création de FO et de la CFDT. Avec les drames de la décolonisation et des répressions en Afrique et en Asie, les propagandes désormais télévisées, selon les préceptes de Le Bon et Goebbels, vont rétablir l'unité nationale. Ici réside peut-être le génie de la mentalité collective française : il n'y aurait jamais de rupture sociale dans l'histoire et ses événements, le " peuple » reste fier et immuable, ce qui n'a rien à voir avec une conscience collective. De beaux parleurs convoquent encore aujourd'hui Vercingétorix, un rebelle, ou Jeanne d'Arc, une bâtarde, pour émouvoir les foules.

8 Les syndicats CGT et CFTC, puis ensuite CGT-FO, en 1954, et CFDT, en 1965 adoptent donc un modèle néo-corporatiste pour organiser socialement les firmes dont la production est indispensable à l'Etat: EDF, Rail, Aviation, EDF, GDF, les PTT (la Poste), l'Education Nationale, les professions portuaires. Quels que soient les parts d'actionnariat de l'Etat, les syndicats évaluent avec la complicité des hiérarchies professionnelles les compétences des salariés, orientent les carrières professionnelles, les mutations et les promotions. De fait, les arguments de fraternité, solidarité, de construire un monde meilleur, de permettre l'accès à l'éducation, la culture, les loisirs, le bonheur, la prise en charge des enfants, vont de pair, avec la notion d'intérêt public, et ceci recouvre les négociations entre acteurs collectifs, entre syndicats de branche, fédérations patronales correspondantes, et l'Etat qui légifère sur les «avantages acquis ». Ces derniers sont obtenus selon les moments et situations par complicité ou proximité politique, poids des menaces qui consiste à arrêter une production stratégique, ou encore par des alliances des salariés avec les fédérations patronales pour externaliser vers le contribuable général des coûts de production particuliers.

9 À la fin des «Trente Glorieuses » et au début de la récession des emplois, une offensive des nouveaux mouvements de droite fonde de nouvelles coordinations entre les Ordres professionnels et des professions à « licence »: taxis, restaurateurs-hôteliers, bureaux de tabac, petits commerçants, artisans, agriculteurs. Le régime des licences professionnelles a été créé sous Vichy, les licences fonctionnent comme l'achat d'une autorisation exclusive d'exercer, d'un capital que l'on peut louer, comme pour les pompes funèbres, les ambulances, les taxis, etc. Les «privilèges» des salariés sont attaqués par ceux qui se revendiquent comme libéraux, mais dont les électeurs sont membres de corporations ou de syndicats professionnels jaloux de leurs prérogatives (leurs caisses de retraite et assurances sociales autonomes sont en faillite). Le paysage politique français se recompose. L'extrême droite prend appui sur les professions à licence, alors que les libéraux, les ancêtres de RPR, UMP, PS sont tenus à détruire les citadelles CGT/PC au nom de la "démocratie». L'exaltation de la fraternité, de la solidarité continue de réunir toutes les classes d'âge dans les corporations jusqu'au début des années 2000.

Les débuts de la $V^{e}$ République s'annoncent, pour beaucoup d'historiens anglo-saxons et d'anthropologues du domaine politique, comme le rétablissement des structures françaises de l'Ancien Régime ${ }^{5}$ : nous avons des corporations strictes organisées par des Ordres et des corporations professionnelles organisées en syndicats professionnels qui sont d'une grande influence politique auprès des élus. Dans le secteur du tourisme par 
exemple, des subventions, donc des impôts d'Etat, (" neige ", " pluie », " sécheresse ", " tempêtes ", selon les climats locaux et annuels) et ils externalisent donc les coûts de production en direction des contribuables. Le néo-corporatisme des professions salariées apparaît bien sage pendant ces années, malgré la programmation et la suppression des emplois miniers (600 000 personnes) entre 1975 et 1985. Le personnel politique et les élus apparaissent alors comme les successeurs des courtisans de l'Ancien Régime : ils n'ont de compte à rendre à personne, pratiquent le nomadisme politique comme on change de salon, s'auto-votent des augmentations d'indemnité de $16 \%$ en 2000, (et plusieurs fois ensuite) lors du passage à l'Euro, alors que les salaires sont encore toujours bloqués. Certains sont parlementaires en France depuis 4 générations, certains salarient femmes, enfants, cousins comme attachés parlementaires, ce qui est passible de prison partout ailleurs qu'en France, mais le Génie Français c'est de légaliser la corruption ${ }^{6}$ (ces propos ont beaucoup intéressé les sociologues africains). En février 2017, le Sénat vote une loi qui prescrit toute délinquance d'activité financière au-delà de 12 ans pour les élus, ce qui exonère les anciennes générations, les réseaux Corses, Marseillais, le BTP et au moins deux candidats actuellement à la présidence. So French !

11 Mais comme nos élus vivent dans des situations hors-sol et hors-temps d'une reproduction heureuse, qui nous coûte quand même 1 million d'Euro/personne, si l'on compte l'entretien des palais, les cuisines, les jardins, la sécurité, nous pouvons comprendre leur grande charge de travail : la plupart d'entre eux en connaissent moins que mes étudiants en matière de finance, industrie, technologie, travail, emploi, environnement. Nous pouvons les soupçonner d'être non seulement incultes, mais paresseux. Vivant dans un monde social, certes actif, mais déconnecté des dynamiques et des inventions de la société concrète, ou seulement de temps en temps, selon leurs curiosités, leurs énergies et leurs intérêts, ils font figure d'un groupement social «à la ramasse », pour employer une expression populaire. Un "mal nécessaire » selon les populations socialement classables souple d'échine, mais d'autres le sont moins...

12 Cet idéal d'un Etat d'autrefois, où toute activité est harmonieuse, perdure, à part les actions de quelques "terroristes", anarchistes, puis Arméniens, Juifs dans les années 1940, puis Algériens vers 1960. Avec les guerres de décolonisation en Europe et aux USA concomitantes avec la guerre froide qui verra la plupart des essais de régimes socialistes et nationalistes détruits en Afrique, une nouvelle catégorie de population apparaît : les " hors Etat », comme l'on désignait ceux qui n'appartenaient à aucune corporation avant 1789 , c'est à dire les chômeurs, les jeunes, les voyageurs, les ambulants, qui sont très vite perçus comme "ennemis de l'intérieur ". Rappelons également qu'au début des années 1970, les pays producteurs de pétrole s'organisent pour imposer leurs tarifs, ce qui se traduit immédiatement par des augmentations de chômage, l'affaire devient sérieuse en France lorsque les cadres sont touchés au début des années 1980. Les mouvements de jeunesse, Beatniks, Hippies, les débuts du « retour à la terre ", l'écologie, les influences maoïstes et libertaires, les mouvements locaux auto-gestionnaires vont constituer un ferment pour les années suivantes. Mais les corporations ou professions protégées, (EDF-GDF, SNCF, Police, Education Nationale, Air France, etc.) comme le personnel politique qui fait la chasse aux indemnités parlementaires, transmettent à leurs enfants les mentalités et cultures professionnelles issues des préceptes du gouvernement de Vichy : on est anti-communiste, puisque tout avantage de salaire, de retraite, de couverture sociale n'est pas transmissible ou généralisable aux autres professions, même si la CGT ou d'autres syndicats négocient 
pour la corporation ou la "branche», on est anticapitaliste, puisqu'on ne saurait tolérer que les outils de production soient vendu à des opérateurs privés. La société de travail française n'a finalement jamais surmonté les dilemmes des tensions entre la CGT et CGTU du début du XXe siècle : les avantages et les conforts arrachés pour une branche professionnelle lors des négociations avec l'Etat se font toujours au nom du «Bien Public", de l'«Intérêt Général» comme s'ils devaient profiter à tous. L'organisation corporatiste est foncièrement inégalitaire : les argumentations ou mises en scènes discursives de la télévision sont-elles encore efficientes aujourd'hui pour stimuler les imaginaires et idéologies d'une société égalitaire ? On pourrait au contraire penser que si l'on met en évidence statistiquement des reproductions professionnelles parents/enfants, comme il est d'usage en sociologie, nous vivons dans une société de caste ${ }^{7}$.

Nous aurions alors une société divisée en quatre parties : $1^{\text {ère }}$, les élus, membres du personnel politique, leurs familles et courtisans; $2^{\text {ème }}$, les ouvriers qualifiés (dits OP aujourd'hui) mais aussi les techniciens de fabrication et techniciens d'atelier, (TF, TA) fondateurs des premiers syndicats au XIXème devenus CGT; $3^{\text {ème }}$, les ouvriers non qualifiés, dits OS, dont on compte énormément de femmes et d'immigrés, auquel on peut rajouter de nombreux intérimaires et CDD, qui ont su, après 1914-18 et les mouvements sociaux du moment, rallier la CGT devenu un temps CGTU (Unifiée) ; ${ }^{\text {ème }}$, les sans-Etat, sans convention collective, dits aujourd'hui également « sans-dent ». Mais il ne s'agit là que de salariés; il faut ajouter à cette typologie, les professions libérales, les professions sous licence et les artisans, agriculteurs, ainsi que les autoentrepreneurs, rêve des mouvements libéraux puisque l'on passe du contrat salarial (une location) à un contrat commercial où selon le montant financier alloué, la personne (ou le client) cotise ou non aux caisses d'assurances maladie et retraite. américains et autrichiens vient à nouveau contrarier ce modèle social heureux pendant les années 1980 alors que les révoltes des "sans-Etat ", des laissés pour compte, jeunes et immigrés font rage dans les banlieues, comme à Vénissieux et Vaulx-en-Velin.

en libérale prend comme argument l'Egalité pour projeter de mettre en concurrence les salaires, couvertures sociales, retraites et conventions collectives de manière supranationale. La chute du mur de Berlin offre une occasion inespérée de faire redoubler ces concurrences en intégrant les ex-pays de l'Est : Pologne, Roumanie, Bulgarie, etc. Des réseaux de centaines de sociologues, d'économistes et de juristes (dont je faisais partie) travaillaient au sein de leurs équipes sur «l'harmonisation » européenne des régimes professionnels, tout en doutant déjà fortement de la possibilité de sa réalisation.

16 Le projet était connu vingt ans avant la mise en place de la Constitution Européenne de 2005, la directive Bolkestein est désavouée par référendum de la part des français mais appliquée par l'UMP et le PS en janvier 2006 par voie parlementaire. Il s'agit d'un typique déni de démocratie et ces partis « de gouvernement » vont ensuite se déliter.

Pendant les années 1990, il est clair que les « partis de gouvernement » UMP-PS avaient déjà divorcé avec les groupes sociaux "sans-Etat", travailleurs étrangers et classes d'âges jeunes avec le projet d'un «SMIC-jeune » en 1995, ce qui va provoquer des remous. Une partie des travailleurs issus de certaines corporations non couvertes par le régime général des assurances sociales et des retraites, comme les agriculteurs, les artisans ou les commerçants, rejoint le Front National, avec une partie des ouvriers 
précarisés, ce qui ne les empêche pas de continuer à adhérer à la CGT pour les salariés ${ }^{8}$. Dans les années 1950/1960, une partie des "indépendants ", sous l'influence des élus Poujade et de Le Pen, a refusé de cotiser au régime général des assurances sociales. Leurs enfants et petits-enfants le regrettent aujourd'hui : comment être couvert juridiquement par les assurances sociales alors qu'on a refusé syndicalement d'y cotiser pendant presque 70 ans?

Pendant cette même période, la diffusion dans la société civile des technologies de la communication comme internet et les mails qui étaient auparavant réservées à l'armée, la police, les Préfectures, les élus, se diffusent au sein des sociétés civiles, ce qui donne naissance à de nouvelles organisations des mouvements sociaux et associatifs. Des exemples connus sont l'invention des tri-thérapies par les associations des malades du sida ou la diffusion de contre-informations des riverains du Morbihan, Loire Atlantique, Vendée lors de la marée noire de l'Erika qui mettent la télévision, les Préfectures et les municipalités en porte à faux ${ }^{9}$. Mais les réseaux deviennent également facilement internationaux avec les mouvements alter-mondialistes, les paysans sans terre du Brésil, les enfants travailleurs en Inde et les premiers forums sociaux mondiaux à partir de 1992 à Davos puis continentaux en Amérique Latine, en Afrique, en Europe. En général, les associations laïques et religieuses, des syndicats et partis politiques, les associations humanitaires, des universitaires, la presse, des ONG, la LDH et d'autres sont présents, les échanges sont intenses entre les différents pays. La création d'ATTAC dans la région des Pays de Loire en 1998 a rassemblé des publics de même composition, étudiants, agriculteurs, médecins, syndicalistes. Un coup d'arrêt à ces mouvements alter-mondialistes qui commençaient à inquiéter le personnel politique international a été porté avec la destruction des tours jumelles à New York en 2001 et avec la mise en place d'un nouvel ordre militaire et policier mondial.

Les résistances sont sourdes ou exprimées lorsqu'elles passent par des coordinations associatives et intersyndicales. Depuis les années 2000, avec le naufrage de l'Erika, le projet de construction de l'aéroport de Notre-Dame-des-Landes (depuis 1962 !), la mise en place des directives européennes comme le mariage pour tous ou la loi «travail», tous les printemps de Loire Atlantique sont émaillés de manifestations. Les jeunes gens ont l'occasion de se rôder à la guérilla urbaine.

Les une après les autres, les branches professionnelles sont attaquées au nom d'un principe d'égalité et de "syndicratie », il s'agit de stimuler les jalousies de statut social et de distraire le peuple. Comme les statuts professionnels solidement encadrés par les juridictions coûtent cher, on favorise depuis les années 90 la mise à son compte, l'autoentreprenariat et le télétravail. Tout d'abord réservé aux secrétaires, ces statuts précaires, car il faut déclarer ses charges sociales, se sont développés chez les ouvriers dans la métallurgie, les bureaux d'études, les industries de la culture, le bricolage, le bâtiment, les aides à la personne : les vieux idéaux de "suppression du salariat » ont été théorisés pendant les années 1990. L'Education Nationale, profitant de l'immense réservoir de main-d'œuvre européen à bas coût a supprimé nombre de formations techniques et manuelles puisque l'on peut aller chercher des compétences autour de la mer Baltique ou de la mer Noire. Les régulations professionnelles dans les corporations s'organisent par la mise en place d'une pénurie, ce qui permet de maintenir le montant des honoraires. Il ne s'agit pas seulement de faire venir des ouvriers agricoles Ukrainiens ou Sénégalais, il y a aussi d'autres catégories touchées comme celle des médecins : on fait venir des médecins roumains pour combler les déserts médiaux dans 
les régions rurales (4000 en Loire Atlantique) et permettre des dépassements d'honoraires en ville.

21 Le Parlement européen remplace la chambre des députés français qui ne produit plus que $20 \%$ des lois votées en France, mais au nom de la démocratie, il faut faire semblant de travailler, alors que des fonctionnaires assermentés pourraient bien signer les lois européennes livrées déjà rédigées pour remplacer nos élus. Il faut également multiplier le nombre d'élus avec l'invention de nouvelles structures comme les palais des régions et des départements ou l'interdiction du cumul des mandats. Nos députés sont également marginalisés par le développement de la gouvernance privée globale, régionale, locale avec les ONG, les fondations, les think tanks, les associations commerciales, les mafias, les paradis fiscaux. Les programmes qui relevaient de l'État providence ont été privatisés et sous-traités aux fondations (comme, Ford, Packard, Rockefeller). L'État est devenu post-souverain et l'isomorphisme qui assurait une cohérence entre les peuples, les territoires et la souveraineté légitime est remis en question. Une bonne dose d'imaginaire est donc requise pour identifier des communautés et des solidarités.

Le corporatisme à la française, foncièrement inégalitaire, est donc attaqué au nom du libéralisme et de l'équité. Ses plus solides bastions, comme les Ordres des médecins ou des notaires, sont concurrencés en Europe. L'appropriation par les populations des technologies informatiques ne permet pas de prévoir si les résistances collectives vont s'affirmer ou si l'individualisme va devenir un modèle obligé.

\section{NOTES}

1. E. Durkheim (1911), «Corporations et morale professionnelle » in « CEuvres complètes », T2, Éditions Minuit, 1954.

2. G. Le Bon (1895 première édition), Psychologie des foules, Flammarion, 2009.

3. C. Maurras (1936), Mes idées politiques, Fayard.

4. J.P. Le Crom (1995), Syndicats nous voilà ! Vichy et le corporatisme, L'Atelier.

5. M. Abeles (2008), Anthropologie de la globalisation, Payot.

6. B. Lefebvre (2012), «C'est la faute à la corruption!» in Ethnographie des travailleurs en déplacement. Voyages en Europe sociale, L'Harmattan.

7. L. Dumont, (1967), Homo Hierarchicus, Gallimard.

8. Il est évident que les délégués syndicaux locaux CGT ainsi que CFDT et FO se refusent à tout commentaire statistique aujourd'hui. Il est également important de préciser que les ambiances syndicales sont extrêmement différentes d'une région à l'autre, voir d'une ville à l'autre, selon les types d'activité de travail.

9. B. Latour, M. Callon (2000), Essai de démocratie technique, La Découverte. 
INDEX

Mots-clés : corporations professionnelles, conformisme français, inégalités sociales, résistances, avenir

\section{AUTEUR}

\section{BRUNO LEFEBVRE}

Anthropologue, sociologue, professeur à l'université de Nantes, membre du LISE-CNRS, Paris. Recherches sur la création de richesses, emplois, travail, entreprises en Europe et en collaboration internationale. 